\title{
ECorregiones de la península de Baja California: UNA SÍNTESIS
}

\author{
Charlotte E. González-Abraham ${ }^{1}$, Pedro P. Garcillán² ${ }^{2}$, Exequiel Ezcurra ${ }^{3,5}$ \\ y el Grupo de Trabajo de Ecorregiones ${ }^{4}$ \\ 1 EcoDesertica, Ensenada, Baja California \\ ${ }^{2}$ Centro de Investigaciones Biológicas del Noroeste \\ ${ }^{3}$ UC MEXUS, University of California, Riverside \\ ${ }^{4}$ Ver integrantes en agradecimientos \\ ${ }^{5}$ Autor para la correspondencia: exequiel@ucr.edu
}

\begin{abstract}
Resumen: La singularidad biológica de la península de Baja California ha capturado el interés de los naturalistas por largo tiempo. Fruto de ello ha sido la publicación de una rica variedad de interpretaciones geográficas de su escenario ecológico y biogeográfico. Aunque coincidentes en un marco común general, presentan también numerosas discrepancias. En este trabajo se revisa exhaustivamente la literatura publicada sobre el tema con el objeto de identificar las fronteras o zonas de transición que presentan mayor diversidad o discrepancia. Se sometió el diagnóstico al examen y discusión de un taller de expertos, el cual contó con el apoyo de un sistema de información geográfica, generando decisiones consensuadas. Los resultados se presentan en un mapa de regiones ecológicas de la península de Baja California como una síntesis del estudio e interpretación sobre las ecorregiones naturales de la península de Baja California.
\end{abstract}

Palabras clave: Baja California, biogeografía, ecorregiones, vegetación,

\begin{abstract}
The biological singularity of the Baja California peninsula has captured the attention of naturalists since long time ago. As result, a rich variety of ecological and biogeographical interpretations have been proposed. They share a common framework, but at the same time show disagreement. An exhaustive review of published literature was done to identify which ecoregions or transition zones show disagreement. We submitted the discordant transition zones to the analysis of regional experts during a workshop, and with the help of a geographical information system we generated decisions by consensus for the majority of discussed zones. Reults are shown in a map of ecoregions of the Baja California peninsula, as a synthesis of the long time effort of study and analysis developed by individuals and institutions on the natural scenario of the Baja California peninsula.
\end{abstract}

Keywords: Baja California, biogeography, ecoregions, vegetation

$\mathbf{E}^{\mathrm{n}}$ 1963, Faustino Miranda y Efraín Hernández Xolocozi publicaron en el Boletín de la Sociedad Botánica de México uno de los artículos que más ha influido en la ciencia de la vegetación en México: "Los tipos de vegetación de México y su clasificación". El estudio utilizaba una aproximación similar a la empleada por Forrest Shreve (1951) en su clasificación de la vegetación del Desierto Sonorense, basada en las características fisonómicas y de las formas de vida de las especies dominantes. Miranda y Hernández X. explicaban que, para su trabajo,

"los tipos de vegetación se han definido fundamentalmente por su fisonomía, derivada a su vez por de la forma de vida (biotipo) de sus especies dominantes. Forma de vida y en consecuencia fisonomía son en cierto modo expresión de los factores del medio, ya sea climático, edáfico o biótico en que un determinado tipo de vegetación o los elementos que lo forman, se desenvuelven" (Miranda y Hernández X., 1963:30).

Unos años antes, y trabajando exclusivamente en el Desierto Sonorense, Forrest Shreve (1951) había utilizado una clasificación de las formas de vida de las plantas para entender y clasificar la vegetación del Desierto Sonorense. A diferencia de la clasificación de Raunkiaer (1934), en boga en Europa en aquellos años y basada en la localización de las 
yemas de renuevo durante el invierno, Shreve comprendió que en las regiones áridas de México y Estados Unidos las formas de vida están determinadas por la manera de afrontar la escasez del agua. Basado en una extensa experiencia de campo, Shreve comprendió que la geografía de las adaptaciones morfológicas y funcionales de las plantas dominantes frente a la sequía delinea la geografía de la vegetación, con tanta precisión como las características florísticas y taxonómicas de los ecosistemas (McGinnies, 1981).

El éxito del trabajo de Miranda y Hernández X. (1963) estuvo basado en su habilidad para extender y ampliar el enfoque fisonómico-florístico empleado por Shreve (1951) para el Desierto Sonorense hacia el reino complejo de las selvas tropicales, y en poder establecer un criterio clasificatorio robusto y consistente a la escala de todo el país. Ambos esfuerzos supieron vislumbrar la importancia de las formas de vida o tipos funcionales para caracterizar las comunidades, un enfoque que posteriormente se desarrolló con la conceptualización de los gremios funcionales (Root, 1967), grupos funcionales (Cummins, 1974), síndromes adaptativos (Stebbins, 1974; Herrera, 1984) o estrategias (Grime et al., 1988).

Desde la publicación del trabajo de Shreve (1951) para el Desierto Sonorense, un gran número de trabajos han sido publicados sobre la extraordinaria geografía ecológica de la península de Baja California. En un artículo anterior (Garcillán et al., 2010) presentamos una revisión histórica de los esfuerzos realizados para cartografiar ecológicamente la península, desde los padres jesuitas hasta los trabajos más recientes de filogeografía, y destacamos los grandes consensos existentes entre los diferentes enfoques. En ese trabajo mostramos cómo las numerosas regionalizaciones biológicas de la península, aunque difieren en límites y unidades, comparten un marco común, delineado por primera vez para la región desértica peninsular por Shreve (1951), y complementado años después por Wiggins (1980) para las partes no desérticas del extremo sur y noroeste peninsular (Figura 1). Este enfoque común captura la doble polaridad ecológica peninsular: (1) un gradiente norte-sur, resultante de la presencia de clima templado en el noroeste y tropical en el sur, con una extensa región árida de transición entre ambas, y (2) un gradiente este-oeste, resultante de la combinación de una cadena montañosa distribuida intermitentemente a lo largo de la península y la influencia de dos masas de agua marina muy distintas (fría en el Pacífico, cálida en el Golfo de California). El resultado de este marco común es la existencia de tres grandes regiones (independientemente de la terminología utilizada por los distintos autores): (1) templada-mediterránea en el noroeste, (2) tropical en el extremo sur, y (3) región árida de transición entre ambos extremos.

Apoyados en esta definición general sobre las grandes fuerzas climáticas y ambientales, y utilizando como base el mapa de Shreve-Wiggins sobre los tipos de vegetación en la península de Baja California (Figura 1) en este traba- jo nos proponemos realizar una síntesis de las principales propuestas de regionalización biológica publicadas para la península de Baja California. Estas propuestas, compartiendo de manera general el marco común referido, presentan diferencias en la localización de las fronteras entre regiones biológicas. La razón de estas discrepancias locales estriba, por un lado, en que las fronteras de cambio entre regiones suele ser gradual por la falta de barreras ambientales o climáticas drásticas y, por el otro, en que distintos grupos de organismos responden o han respondido de forma distinta a los cambios ambientales, climáticos o ecológicos actuales y del pasado (Lomolino et al., 2006). Asimismo, las diferencias entre propuestas de regionalización se derivan de la multiplicidad de objetivos, datos base, metodologías, o escala espacial empleados por los distintos autores. Lejos de ser un inconveniente, esta diversidad de propuestas es el resultado de aproximaciones diversas basadas en los distintos niveles de organización ecológica y biogeográfica de la región, y sus diferencias son altamente informativas.

Nuestro objetivo en el presente trabajo es identificar las

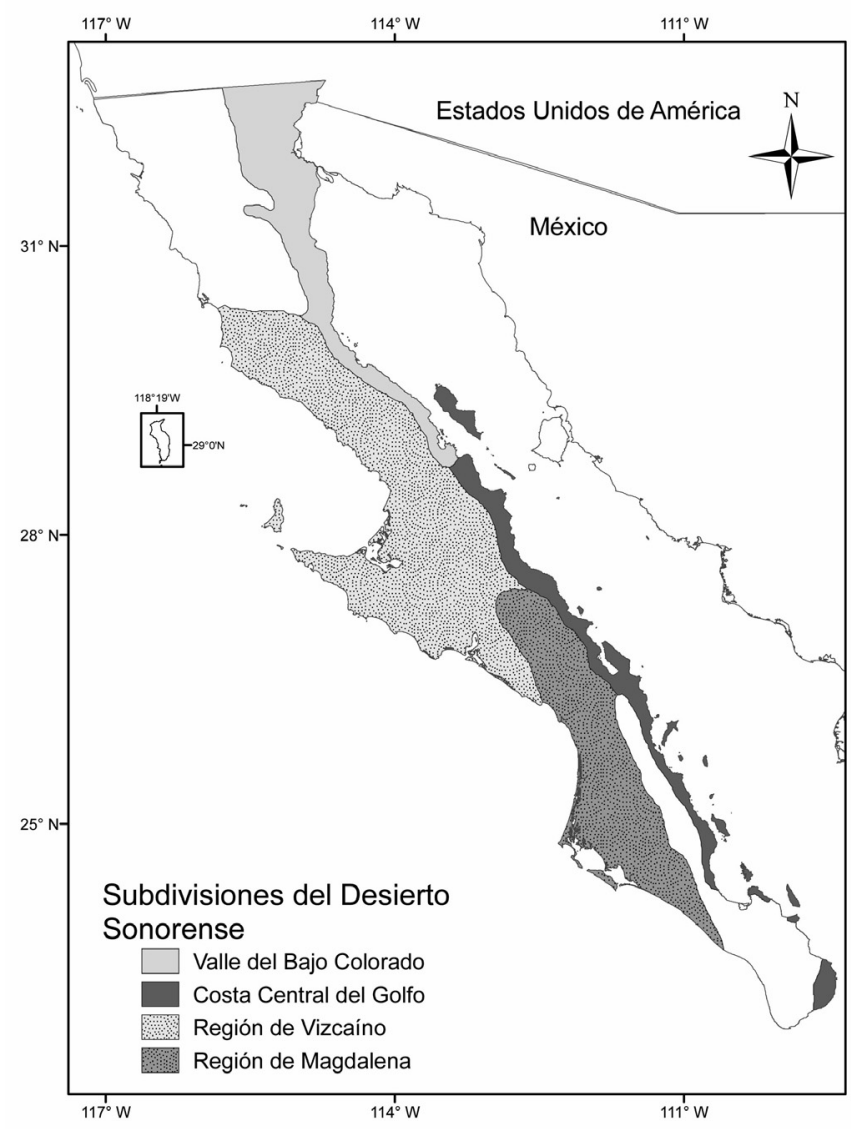

Figura 1. Subdivisiones del Desierto Sonorense presentes en la península de Baja California (Shreve 1951) mostrando el marco biogeográfico común a la mayoría de las propuestas de regionalización peninsular. En blanco se muestran las regiones no desérticas: mediterránea en el noroeste y tropical en el sur. 
principales zonas de discordancia entre las distintas regionalizaciones propuestas para la península de Baja California y precisar en lo posible la compatibilidad y congruencia entre las mismas, para definir al final un mapa de las regiones ecológicas y/o grandes tipos de vegetación en la península de Baja California con el mayor consenso posible.

\section{Métodos}

Como un primer paso a este trabajo, revisamos exhaustivamente la literatura sobre regionalizaciones biológicas de la península de Baja California, y siguiendo la secuencia de su desarrollo histórico identificamos el marco común compartido y las principales zonas de discordancia. En este análisis (publicado en Garcillán et al., 2010), encontramos una gran concordancia entre las diferentes regionalizaciones ecológicas y biogeográficas hechas para la península, desde las realizadas por exploradores y naturalistas usando observaciones de campo sobre las formas de vida dominantes, pasando por estudios basados en florística, hasta trabajos recientes basados en métodos cuantitativos de análisis multivariado y/o métodos moleculares de filogeografía. Encontramos asimismo algunas zonas de variación biogeográfica gradual, en las que se presentaban discrepancias en el establecimiento de límites entre las ecorregiones.

Con el propósito de profundizar en la comprensión y análisis de estas zonas de discrepancia construimos un sistema de información geográfica con información de los siguientes atributos geográficos para toda la península: (a) uso de suelo y tipo de vegetación (INEGI, 1997), (b) precipitación y temperatura (Hijmans et al., 2005), (c) suelos (INEGI, 1995), (d) geología (INEGI, 2004), (d) topografía (INEGI, 1998), y (e) fisiografía (INEGI, 1990). Posteriormente realizamos una extensa revisión de la información climática, geológica y biológica publicada sobre la región, que nos ayudara a profundizar en la naturaleza de las zonas de discordancia.

En este punto del diagnóstico y análisis de las zonas de discordancia convocamos a un taller de expertos regionales para someter a su consideración el diagnóstico tentativo de las áreas de discrepancias y perfilar respuestas de consenso sobre las mismas. Después de analizar la literatura disponible, decidimos colectivamente partir de las regiones de vegetación y la nomenclatura propuestas en el trabajo clásico de Shreve (1951) y revisar la concordancia de esta regionalización con estudios más modernos realizados bajo otros, y diferentes, enfoques metodológicos (Omernik, 1987; Bailey, 1983).

Para analizar las discrepancias y trazar límites de consenso, usamos (a) la información existente sobre las ecorregiones, tipos de vegetación, y biogeografía de la península, obtenida a través de una amplia y exhaustiva recopilación bibliográfica realizada antes del taller; (b) las bases de datos accesibles sobre distribución de especies (Turner et al., 1995; la página web de The Flora of Baja California, San
Diego Natural History Museum; leguminosas leñosas, Garcillán y Ezcurra, 2003); y (c) las notas de campo y conocimiento directo de los participantes en el taller. El trabajo del taller se sustentó sobre el análisis y discusión de los factores y patrones que se describen a continuación.

Análisis de patrones biogeográficos. La vegetación es un integrador del clima, suelo, geomorfología e historia ambiental, y su fisonomía dominante, junto con los datos de distribución geográfica de las especies indicadoras, sirvieron como el criterio fundamental de reconocimiento de fronteras regionales. Por ejemplo, la distribución hacia el sur de la Provincia Florística Californiana se asocia a la presencia de especies rosetófilas capaces de captar niebla y almacenar su humedad (nebulofitas como Agave shawii y Duddleya spp.). Por ello, la distribución del matorral rosetófilo costero en la carta de vegetación de INEGI, sumada a la distribución de las colectas de estas especies indicadoras en las bases de datos, fueron el criterio fundamental para establecer los límites de esta región. De la misma forma, los límites del Desierto Central se asocian a la distribución de la vegetación sarcocaule, dominada por especies de tallos engrosados, como el cirio (Fouquieria columnaris) y el copalquín (Pachycormus discolor). Nuevamente, la distribución de estas especies en las bases de datos florísticas y la distribución del matorral sarcocaule en la carta de INEGI fueron el criterio fundamental para establecer los límites de esta ecorregión. Así, los mapas de vegetación, las bases de datos de distribución de especies y la información publicada sobre patrones filogeográficos, proporcionaron la información necesaria para identificar zonas de endemismo, transiciones entre distintos grupos de especies, o patrones biogeográficos crípticos resultado de la historia geológica, variación en el nivel del mar, o los efectos de los ciclos glaciales del Pleistoceno.

Análisis de factores abióticos. A escala regional, el clima, como fuente de energía y humedad, funciona como el factor causal primario de los ecosistemas (Bailey, 2004), y el patrón general climático es modulado por las características y singularidades de la superficie terrestre. Partiendo de estos puntos, utilizamos como factores causales en nuestras decisiones la información climática (p. ej., Hastings y Turner, 1965; Hijmans et al., 2005) en conjunción con otros atributos abióticos como la fisiografía, topografía y geología. La superposición de estos mapas (clima, geología, fisiografía, y topografía) nos permitió identificar regiones donde el ambiente abiótico cambia de manera intensa, y utilizamos estas zonas de transición para delimitar e interpretar límites ecológicos. Por ejemplo, la transición entre la Provincia Florística Californiana y el Valle del Colorado en el Desierto Sonorense está dada por la sombra orográfica de las Sierras de Juárez y de San Pedro Mártir, y la divisoria de aguas de estas montañas sirvió como criterio fundamental para cartografiar la transición entre estas dos regiones biogeográficas. 
Escala espacial de patrones y procesos. La identificación de fronteras ecorregionales de manera jerárquica implica la comprensión e identificación de los procesos generadores que operan a diferentes escalas (Bailey, 2004). Durante el proceso de análisis y discusión en el taller tuvimos en especial consideración la escala de los procesos biogeográficos, y asumimos, de acuerdo con Rowe y Sheard (1981), que los mapas ecológicos son hipótesis que pueden ser contrastadas y mejoradas. Para ello, examinamos si las observaciones y patrones realizados o publicados por los participantes del taller a nivel micro-geográfico eran consistentes con observaciones hechas y patrones observados a escalas mayores, y a la inversa, si las grandes tendencias observadas a nivel macro-geográfico podían ser puestas a prueba localmente y ver cuán robustas eran sus predicciones. Como ejemplo de este criterio, los límites biogeográficos para la Región del Cabo planteados por León de la Luz et al. (2000) basados en criterios florísticos y geológicos a escala local fueron comparados contra criterios a escala mayor, como la carta de vegetación de INEGI, cartas geológicas de toda la península, o la distribución geográfica de especies de afinidad tropical, y, después de verificar la congruencia entre escalas fueron aceptados como los límites a utilizar en este trabajo.

\section{Resultados y discusión}

Zonas de discrepancia. Identificamos las siguientes zonas de discrepancia principales (Figura 2): (a) transición nortesur entre la región mediterránea y el desierto, (b) sub-regionalización dentro de la región mediterránea, (c) dimensión mediterránea de las grandes islas del Pacífico norte, (d) transiciones en los desiertos costeros del Golfo de California, (e) la compleja transición en el centro peninsular, (f) singularidad de la Sierra de la Giganta, y (g) transición entre la Región del Cabo y el desierto.

Transición norte-sur entre región mediterránea y región desértica.- La mayoría de los estudios proponen el límite entre la región mediterránea y el desierto en algún punto a lo largo del área de transición alrededor de El Rosario (29 $-30^{\circ}$ N), excepto Arriaga et al. (1997) que establecieron el límite norte del desierto en la península de Punta Banda, al sur de la ciudad de Ensenada ( $\left.31^{\circ} 40^{\prime} \mathrm{N}\right)$.

La localización de esta zona de transición ha estado discutida desde el comienzo de los estudios de vegetación sobre la península. Las especies de chaparral se extienden hacia el sur hasta donde disponen de humedad adecuada (Shreve, 1936), en tanto que el alcance septentrional de las especies propias del desierto parece depender de la ausencia de heladas y existencia de precipitación de verano, aunque sea en cantidad mínima (Raven y Axelrod, 1978). Westman (1983) identificó una comunidad de matorral en los alrededores de El Rosario $\left(30^{\circ} \mathrm{N}\right)$, que denominó matorral costero suculento Vizcaíno, y que ubicó dentro de la región Mediterránea aunque reconoció la posibilidad de una mayor afinidad con el Desierto Sonorense. Sin embargo, Zippin y Vanderwier (1994) consideraron que la afinidad, aunque débil, de esta asociación era con el Desierto Sonorense.

Peinado et al. (1995) estimaron que la transición entre el matorral mediterráneo y el desértico se produce entre los paralelos $30^{\circ}$ y $31^{\circ} \mathrm{N}$. Mellink (2002), con base en la distribución de tetrápodos endémicos concluye que el límite sur de la región Mediterránea coincide con el Arroyo del Rosario (aprox. $30^{\circ} \mathrm{N}$ ), y sugiere la existencia de una larga zona transicional entre la ciudad de Ensenada ( $\left.31^{\circ} 52^{\prime} \mathrm{N}\right)$, que sería el límite norte de algunas especies desérticas, y el Arroyo del Rosario, límite sur de los taxa mediterráneos. Álvarez-Castañeda et al. (1995) ubican la transición a la altura de San Quintín ( $31^{\circ} 30^{\prime}$ N), como resultado de su análisis de distribución de mamíferos.

Sin embargo, Epling y Lewis (1942) mostraron cómo la distribución de numerosas especies de plantas característi-

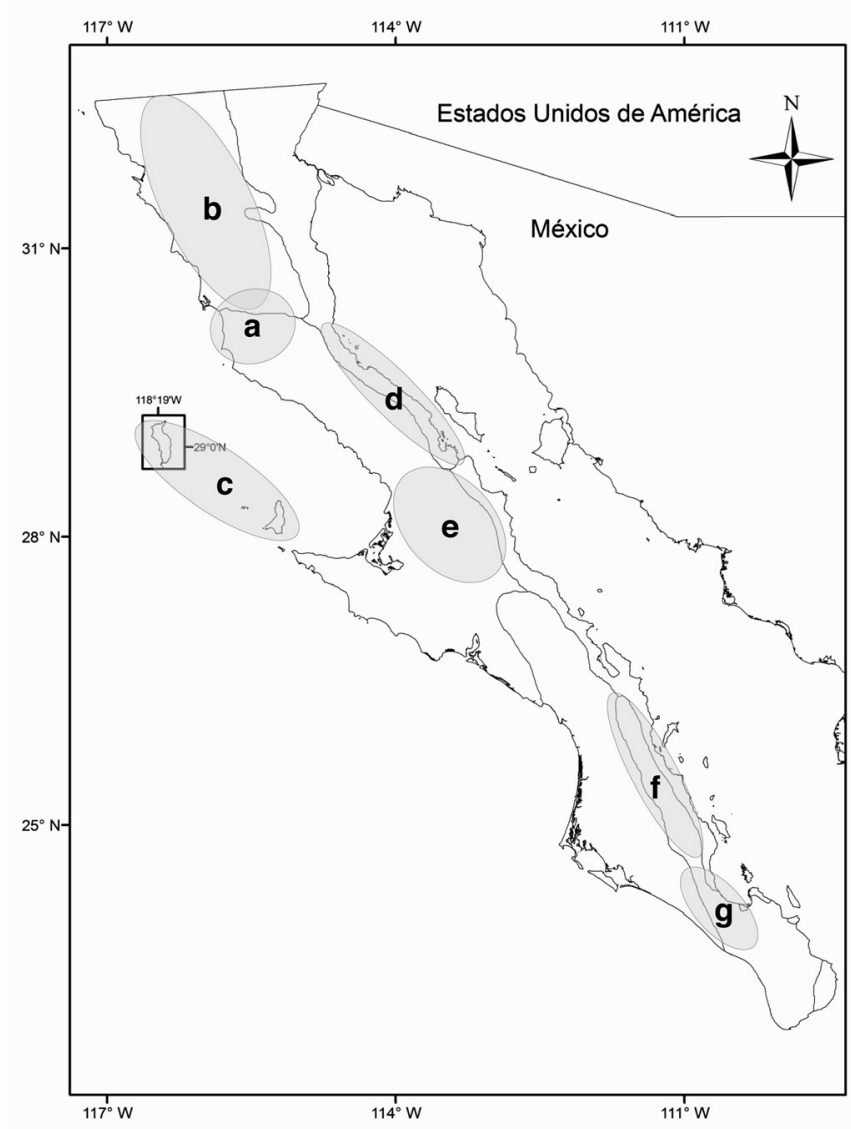

Figura 2. Principales zonas de discrepancia en las regionalizaciones biológicas de la península de Baja California: (a) transición región mediterránea a desértica, (b) sub-regionalización mediterránea, (c) grandes islas del Pacífico Norte, (d) transición de los desiertos costeros del Golfo de California, (e) transición del centro peninsular, (f) singularidad de la Sierra de la Giganta, y (g) transición región tropical a desértica. 
cas de la región Mediterránea se extiende hacia el sur a lo largo de la costa Pacífica. Delgadillo (1998) en su completo estudio sobre la florística de la región propone el límite sur de la vegetación mediterránea aproximadamente a $29^{\circ} 40^{\prime}$ N). Más recientemente, Peinado et al. (2008), con base en un ingente esfuerzo de campo, encuentran una similar prolongación de la vegetación costera de carácter mediterráneo hacia el sur de $\operatorname{los} 30^{\circ} \mathrm{N}$. En nuestra opinión, la distribución de especies de carácter mediterráneo como muestran estos autores es un indicio de que el componente mediterráneo de esta zona de transición se extiende más al sur de los $30^{\circ}$ $\mathrm{N}$, condicionado fundamentalmente por el efecto de la proporción de lluvia de invierno y por un factor poco estudiado aún en la península como es el efecto de la niebla costera en la distribución de la vegetación. Elegimos como criterio de ubicación del límite sur de la región Mediterránea con base en el contorno de porcentaje de lluvia de invierno superior a $70 \%$, en combinación con la topografía. En cierta medida esta transición mediterráneo/desierto se reproduce a menor escala y en un gradiente altitudinal en las dos grandes islas del Pacífico norte: Guadalupe y Cedros como se muestra más adelante.

Sub-regionalización mediterránea.- La delimitación de las sub-regiones dentro de la región mediterránea ha sido diversa. Mientras Murphy (1983) propuso un área continua basado en distribución de herpetofauna, Wiggins (1980) y Zippin y Vanderwier (1994) diferenciaron dos áreas de vegetación en forma de dos franjas paralelas a la costa: región Mediterránea, en la parte de menor altitud, y bosque de coníferas, a mayor altitud, similares a los distritos faunísticos de Nelson (1921). Rojas-Soto et al. (2002) en su análisis basado en la distribución potencial de aves obtuvieron dos franjas similares a las anteriores, pero con la franja costera dividida a su vez en dos que denominaron regiones Diegana Norte y Sur, respectivamente. Además diferenciaron las cumbres altas de Sierra de Juárez como Sierras del Norte de Baja California.

En la clasificación fitosociológica de la península realizada por Peinado et al. (1994a), estos autores definieron dos provincias dentro de esta región: Californiana Meridional y Martirense, con dos sectores dentro de esta última, Juarezense y Martirense. Es interesante que sus resultados reflejaran diferenciación entre provincias y sectores en sentido norte-sur, y no en sentido este-oeste (altitudinal). La regionalización de Arriaga et al. (1997) sólo incluyó dos áreas diferentes: (1) chaparral-matorral costero, ocupando casi en su totalidad la región mediterránea, y (2) dos pequeñas áreas de bosque de pino-encino en las cumbres de Sierra de Juárez y Sierra de San Pedro Mártir, respectivamente. Es necesario recordar la diferente escala geográfica de esta última propuesta, realizada para el conjunto de la República Mexicana.

Mooney y Harrison (1972) encontraron alrededor de $31^{\circ}$
$\mathrm{N}$ de latitud una transición altitudinal de la vegetación, de matorral costero con suculentas en el cinturón de baja altitud $(0-400 \mathrm{~m})$, hacia matorral costero a altitudes intermedias (400-700 m), y finalmente, matorral siempreverde por encima de $700 \mathrm{~m}$ de altitud. En su análisis de los matorrales xerófitos mediterráneos de California y Baja California, Westman (1983) encontró dos transiciones latitudinales en el norte de Baja California. La primera, situada al sur de Punta Banda ( $\left.31^{\circ} 40^{\prime} \mathrm{N}\right)$, tiene lugar entre el matorral costero en el norte (dominado por especies mesófilas y dimórficas estacionalmente) y el matorral costero suculento (especies suculentas y completamente caducifolias) al sur del anterior. La segunda transición ocurre en el interior del matorral costero suculento a la altura de San Quintín (30 30' N) donde define a su vez dos asociaciones: Martirense (entre San Quintín y Punta Banda), equivalente al matorral costero suculento de Mooney y Harrison (1972), y Vizcaína (entre El Rosario, $30^{\circ} \mathrm{N}$, y San Quintín) a la que consideró transicional hacia el matorral desértico. Peinado et al. (1995) en un estudio similar encontraron tres asociaciones: chaparral, matorral costero y matorral costero suculento dentro de los matorrales de la región Mediterránea, coincidentes con lo encontrado por Mooney y Harrison (1972) y Westman (1983).

Finalmente, Minnich y Franco-Vizcaíno (1988) publicaron un detallado mapa de vegetación y uso de suelo de la región Mediterránea, que un tiempo después sintetizaron en un mapa de siete tipos generales de vegetación (Minnich y Franco-Vizcaíno, 1999). La disposición geográfica de estos tipos de vegetación es altamente concordante con las áreas bioclimáticas obtenidas por Peinado et al. (1994a).

Consideramos que la regionalización de Minnich y Franco-Vizcaíno (1988, 1999), similar a Peinado et al. (1994a), engloba y completa los estudios previos y es satisfactoria en tanto que refleja acertadamente el marcado efecto de la modulación de la altitud sobre temperatura y precipitación, así como la fisonomía de la vegetación.

Grandes islas del Pacífico Norte.- Las islas de Cedros y Guadalupe constituyen una variante insular de la transición área mediterránea - desierto. El carácter parcialmente mediterráneo de estas islas ha sido reconocido desde los primeros estudios faunísticos y florísticos realizados en ellas. Aunque Guadalupe ha estado ausente en la mayoría de las regionalizaciones, excepto en Nelson (1921), Bancroft (1926), y Grinnell (1928), su singularidad biológica y biogeográfica es incuestionable. Rzedowski (1978) en su trabajo clásico sobre la Vegetación de México, la considera como una provincia florística distintiva dentro de la Región Pacífica Norteamericana, Reino Holártico.

Epling y Lewis (1942) sugieren su afinidad mediterránea mostrando cómo la distribución de algunas especies del chaparral o del matorral costero se extiende hasta Guadalupe (4 especies) o hasta Cedros (10 especies). Posteriormente, estudios de las floras de musgos de isla Guadalupe, isla 
Cedros y el norte peninsular mostraron nuevamente su afinidad con la Provincia Florística de California (Koch y Crum, 1950; Crum y Miller, 1956). La misma interpretación hacen Smith et al. (1990), que consideraron a Guadalupe como parte de la región Californiana en su análisis sobre la distribución de los moluscos terrestres de la península. Posteriormente, varios autores confirmaron ampliamente la afinidad mediterránea de Cedros (Oberbauer, 1993) y Guadalupe (ej. Thorne, 1969; Wallace, 1985; Moran, 1996). Moran (1996) consideró a Guadalupe como parte de la Provincia Florística de California, ya que comparte gran número de especies con el sur de California y norte de Baja California, y especialmente con las Channel Islands del sur de California.

En nuestra opinión estas dos grandes islas tienen un importante carácter mediterráneo determinado por la combinación de una significativa proporción de lluvias de invierno (muy clara en isla Guadalupe) y un determinante efecto de las nieblas costeras potenciado por su elevada topografía (Guadalupe: 1,298 m, Cedros: 1,194 m). Por esta razón, proponemos que ambas islas se consideren como una ecorregión diferenciada.

Transición en los desiertos costeros del Golfo de California.- Shreve (1951) fue el primero en defender la completa "separación" de la vertiente de la costa del Golfo respecto de la del Pacífico, consecuencia de la combinación de los efectos marinos diferenciados en ambas costas separados por una barrera montañosa intermitente de norte a sur.

La transición entre los desiertos en la costa del Golfo ha sido localizada en dos zonas diferentes: alrededor de la Bahía de San Luis Gonzaga (29 45’ N) (ej., Murphy, 1983; Peinado et al., 1994a; Arriaga et al., 1997) o ligeramente más al sur, a la altura de Bahía de los Ángeles (29 N) (ej., Nelson, 1921; Shreve, 1951; Wiggins, 1980; Faulkner, 1982; Brown et al., 1992; Brown, 1994; Zipping y Vanderwier, 1994; Álvarez-Castañeda et al., 1995), o incluso una doble transición (Rojas-Soto et al., 2003). Probablemente esta dicotomía es debido a la presencia de dos zonas de transición escalonadas o quizá una extensa transición como muestran la distribución de herpetofauna (Grismer, 1994, 2002) o plantas de la familia Acanthaceae (Daniel, 1997) o Leguminosae (Garcillán et al., 2003). Ante la falta de argumentos determinantes, optamos por mantener los límites propuestos por Shreve (1951), ajustándolos aproximadamente a la cuota altitudinal de $350 \mathrm{~m}$, desde el sur de San Pedro Mártir hasta Bahía de los Ángeles.

Transición centro-peninsular.- Esta zona de transición, al menos parcialmente asociada a la existencia de un brazo de mar recientemente apoyado por análisis filogeográficos (ej., Upton y Murphy, 1997; Aguirre-L. et al., 1999; Riddle et al., 2000; Zink, 2002) fue genialmente intuida por el Padre Jesuita Miguel del Barco (1780), cuando interpretó la existencia de conchas marinas en el interior peninsular lejos de la costa como resultado de la elevación de una parte de la superficie previamente sumergida. La transición entre el componente tropical y no tropical de la biota peninsular fue tempranamente identificada por los primeros naturalistas y situada entre las latitudes de $27^{\circ}-28^{\circ} \mathrm{N}$ (Brandegee, 1892; Eisen, 1900; Nelson, 1921).

Aunque Nelson (1921) y Shreve (1951) reconocieron el carácter particular de las llanuras del Desierto de Vizcaíno (de hecho el nombre fue acunado por Nelson), ambos lo incluyeron sin diferenciar como parte de sus extensas regiones centrales (Distrito del Desierto de Vizcaíno y Región de Vizcaíno, respectivamente). Aschmann (1959) consideró que en esta gran región central se debía diferenciar dos regiones: Desierto Central (al norte) y Desierto de Vizcaíno (al sur), siguiendo aproximadamente el contorno de la línea de $100 \mathrm{~m}$ de altitud, basado en el clima (exposición al viento y temperatura atenuada por el efecto de la brisa marina) y la vegetación. Gentry (1978), en su muy sugerente y pionera interpretación de las relaciones entre la distribución y la evolución de las especies del género Agave con los cambios geológicos de la tierra en Baja California, sugirió la existencia de un brazo de mar en el pasado apoyado en la separación morfológica y anatómica de los complejos de Agave sobria y A. cerulata.

Entre los estudios revisados podemos distinguir propuestas que definen una zona de transición norte-sur en el centro peninsular (ej., Murphy, 1983; Rojas-Soto et al., 2003; Garcillán y Ezcurra, 2003) y otras que no proponen una transición explícita (ej., Wiggins, 1980; Peinado et al., 1994a; Zipping and Vanderwier, 1994; Arriaga et al., 1997).

En nuestra opinión en la región central de la península tiene lugar la transición del área peninsular con lluvias predominantemente veraniegas (sur y este) e invernales (costa noroeste), con la impronta biogeográfica generada por la existencia de un brazo de mar (Riddle et al., 2000), por lo que constituye una zona de compleja transición norte-sur en la distribución de múltiples grupos de especies (Turner et al., 1995; Álvarez-Castañeda y Patton, 1999; Grismer, 2002; Zink, 2002). Decidimos establecer las fronteras entre el Desierto Central y el Desierto de Vizcaíno con base en el cambio topográfico que ocurre entre ambas regiones, en combinación con la distribución del cirio (Fouquieria columnaris), que expresaría las fronteras del Desierto Central.

Singularidad de la Sierra de la Giganta.- Shreve (1951) consideró la región de la Sierra de la Giganta y la Región del Cabo como no pertenecientes al Desierto Sonorense. Wiggins (1980) propuso dos regiones generales dentro de la gran Región del Cabo: Región de la Sierra de la Giganta y Región Tropical Árida. El análisis de Zipping y Vanderwier (1994) apoya esta frontera entre la vegetación del septentrional brazo alargado de la región subtropical y la muy distintiva vegetación de la Región del Cabo. Sin embargo, estos autores señalaron que la separación de la vegetación de la Sierra de la Giganta del Desierto Sonorense era una cuestión 
aún por resolver. Brown et al. (1992) en sus análisis sobre la distribución de las mariposas peninsulares establecen una diferenciación similar entre la Región del Cabo y la región de la Sierra de la Giganta, que ellos denominaron Provincia de la Región del Cabo y Provincia Extendida del Cabo. Sin embargo, la Sierra de la Giganta no aparece diferenciada en el análisis ornitológico de Rojas-Soto et al. (2003).

Recientemente, León de la Luz et al. (2008) utilizaron análisis florísticos y biogeográficos para mostrar que la flora de la Sierra de la Giganta es compartida parcialmente con la vegetación tropical de las tierras bajas de la Región del Cabo, pero también con las montañas presentes en el desierto del centro peninsular. Concluyen que la flora de $\mathrm{La}$ Giganta es parte de un continuo a lo largo de las montañas volcánicas del sur de la península y podría ser considerada como una nueva región. Con base en ello consideramos la Sierra de la Giganta, sensu extenso, y la definimos en base al factor topográfico, siguiendo aproximadamente la altitud de $500 \mathrm{~m}$, en el conjunto de sierras que van desde Cerro el Mechudo en el sur $\left(24^{\circ} 45^{\prime} \mathrm{N}\right)$ hasta el Volcán de las Tres Vírgenes $\left(27^{\circ} 30^{\prime} \mathrm{N}\right)$.

Transición Región del Cabo - Desierto Sonorense.- La Región del Cabo es el área más distintiva de toda la península y ha sido reconocido reiteradamente en todos los estudios de dimensión geográfica, desde los estudios de los primeros naturalistas (Eisen, 1897; Brandegee, 1891, 1892) hasta los más recientes análisis filogeográficos (Aguirre-L. et al., 1999; Riddle et al., 2000; Zink, 2002). La ubicación del límite de esta transición ha sido sin embargo localizada en diversas zonas del istmo de La Paz (Murphy, 1983; Peinado et al., 1994a; Zippin y Vanderwier, 1994; León de la Luz et al., 2000; Garcillán y Ezcurra, 2003; Rojas-Soto et al., 2003). Arriaga et al. (1997), sin embargo, establecieron el límite occidental de la Región del Cabo a lo largo de una línea aproximadamente en sentido norte-sur que une la ciudad de La Paz con Todos Santos. León de la Luz et al., (2000) propusieron que la frontera que separa los sedimentos de origen granítico de los sedimentos de origen basáltico debería ser usada como un atributo paisajístico fácilmente identificable para delimitar la transición.

Esta propuesta de límites reúne la coincidencia de dos discontinuidades ecológicamente transcendentales, el sustrato geológico y un cambio significativo en la precipitación anual. Finalmente, la subregionalización dentro de la Región del Cabo fue basada en la caracterización progresiva que partiendo de Shreve (1937), han realizado Arriaga y Ortega (1988), Arriaga y León de la Luz (1989), León de la Luz y Domínguez-Cadena (1989) y León de la Luz et al., (2000).

Propuesta sintética de regiones ecológicas. Definimos un total de 14 ecorregiones agrupadas en tres grandes regiones: Región Mediterránea (5), Desierto (6) y Región del Cabo (3) (Figura 3).
Región mediterránea (Provincia Florística de California).Constituye una de las dos regiones no desérticas de la península de Baja California. Está situada en el noroeste peninsular y contiene el extremo sur de la Región Mediterránea Californiana o Provincia Florística de California. Tiene dos periodos confiables de precipitación, invierno y primavera (Hastings y Turner, 1965). Dentro de esta región, pueden definirse varias ecorregiones basadas principalmente en altitud, precipitación e influencia marítima.

La descripción de está región está basada en Shereve (1936), Epling y Lewis (1942), Thorne (1969), Mooney y Harrison (1972), Wiggins (1980), Westman (1983), Wallace (1985), Oberbauer (1993), Zippin y Vanderwier (1994), Peinado et al. (1994a, 1994b, 1995, 2008), Moran (1996), Delgadillo $(1998,2004)$ y Minnich y Franco-Vizcaíno (1998, 1999).

(i) Sierras de Juárez y San Pedro Mártir-- Situada en las montañas del norte de Baja California, esta ecorregión es continuación de la porción sur de las Cadenas Montañosas Transversales y Peninsulares en el sur de California. Contiene dos cadenas montañosas, la Sierra de Juárez y la Sierra de San Pedro Mártir, con alturas máximas de 1,200 m y 3,100 m, respectivamente, y un descenso vertiginoso en su flanco oriental hacia el valle del Bajo Colorado. En la vertiente oeste, en cambio, desciende más suavemente hacia el Océano Pacífico y entra en transición gradual con la ecorregión del Chaparral.

El clima es de tipo mediterráneo con inviernos fríos y lluviosos, y veranos secos y cálidos, con ocasionales tormentas. La precipitación anual fluctúa entre 500 y 700 mm, superior a cualquier otra ecorregión del norte peninsular. Alrededor del 75\% de la precipitación está concentrada entre Octubre y Abril. La nieve en Sierra de Juárez representa sólo el 25\% del total de la precipitación, pero en San Pedro Mártir, por encima de 2,200 m puede alcanzar el 50\%.

Estas montañas constituyen, junto con la Sierra de La Laguna en el sur, los únicos paisajes forestales de toda la península. Pinus y Abies son los géneros dominantes fisonómicamente. Contiene masas de Pinus quadrifolia y $P$. jeffreyi, el primero es dominante en la Sierra de Juárez, acompañado por $P$. monophylla, mientas que el pino Jeffrey es dominante en las altas cimas de la Sierra de San Pedro Mártir, especialmente entre 1,500 y 2,000 m, en masas mixtas con Pinus lambertiana y Abies concolor. Arbustos frecuentes son las manzanitas (Arctostaphylos pringlei y A. pungens), Quercus peninsularis, y Salvia pachyphylla. Bosques de Populus tremuloides se encuentran en los lugares especialmente húmedos a través de San Pedro Mártir por encima de $2,300 \mathrm{~m}$.

(ii) Chaparral.- El chaparral es una comunidad siempreverde de arbustos esclerófilos que cubre el piedemonte de las Sierras de Juárez y San Pedro Mártir, hasta los 1,800 m. Desciende hasta elevaciones de 400-600 m, donde es re-emplazado por el matorral costero. Está frecuentemente aso- 


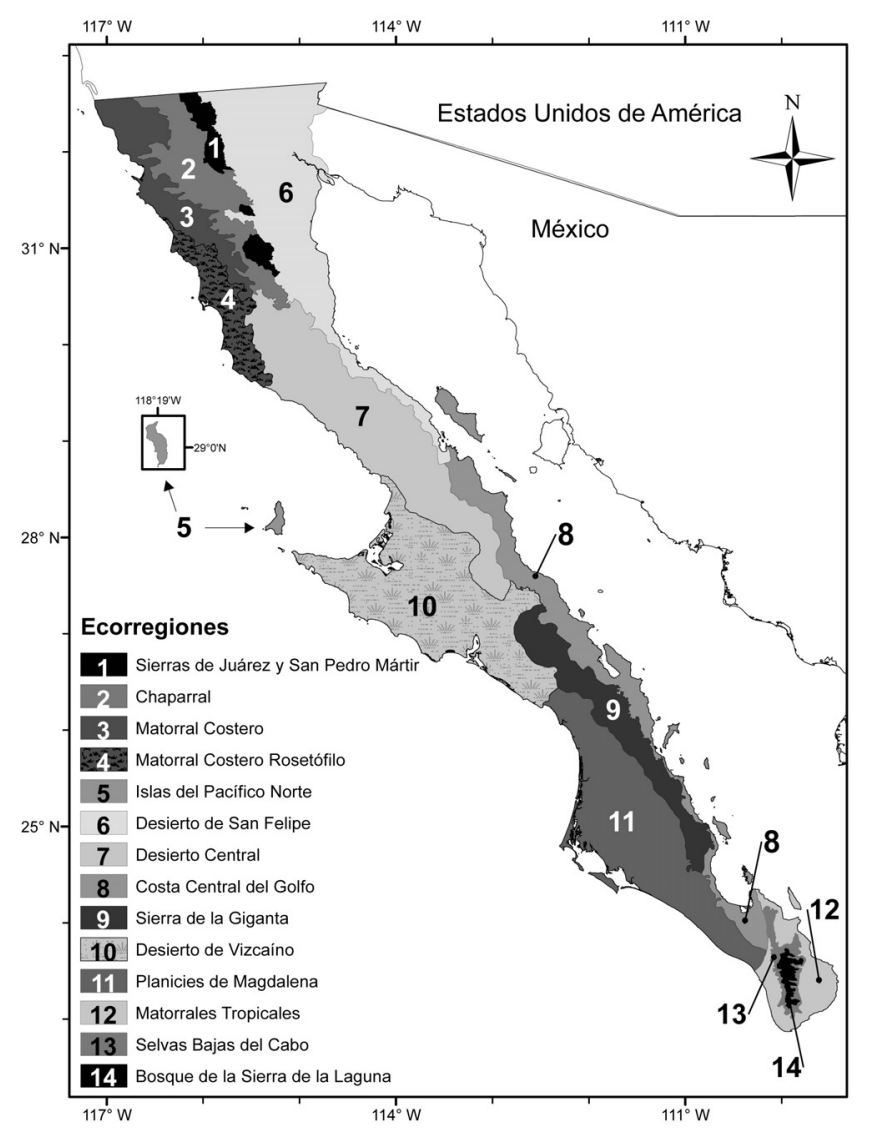

Figura 3. Propuesta sintética de ecorregiones de la península de Baja California.

ciado con suelos de baja fertilidad, de textura gruesa, y una composición variable de especies dependiendo de la altitud y orientación.

La precipitación anual varía con la elevación, variando entre $160 \mathrm{~mm}$ y $500 \mathrm{~mm}$. Las lluvias invernales son la principal fuente de precipitación y en las partes más altas pueden ocurrir ocasionales tormentas de verano. A baja altitud, en cambio, los veranos son cálidos y secos con temperaturas que alcanzan frecuentemente $\operatorname{los} 38^{\circ} \mathrm{C}$ y alto riesgo de incendios. Los inviernos son moderados a baja altitud, pero en las partes más altas las heladas no son infrecuentes.

El chaparral es un matorral denso, de un solo estrato de 1-3 m de altura, compuesto por arbustos de ramas rígidas con hojas esclerófilas de reducido tamaño y extensos sistemas radiculares. La composición de especies del chaparral varía según la altitud y orientación. Está caracterizado por la amplia presencia del chamizo de vara prieta (Adenostoma fasciculatum) y del chamizo de vara roja (Adenostoma sparsifolium), acompañadas por una mezcla variada de especies de los géneros Ceanothus, Arctostaphylos y Quercus. Otros arbustos comunes son Malosma laurina, Cercocarpus betuloides, Xylococcus bicolor, Rhus ovata, Heteromeles arbutifolia, Rhamnus ilicifolia, Ornithostaphylos oppositifolia,
Fraxinus parryi, Aesculus parryi, Eriophyllum confertiflorum y Gutierrezia sarothrae.

(iii) Matorral costero.- En Baja California el matorral costero sigue la costa Pacífica desde la frontera internacional hasta San Vicente ( $\left.31^{\circ} 20^{\prime} \mathrm{N}\right)$. Al sur de este punto, da lugar a un cinturón de transición entre el matorral costero suculento y el chaparral hasta la latitud de $30^{\circ} 20^{\prime} \mathrm{N}$. El matorral costero se encuentra desde el nivel del mar hasta elevaciones de 400-600 m, cubriendo las colinas costeras y las pendientes bajas de las montañas. El paisaje se caracteriza por acantilados marinos, terrazas fluviales y costeras, y dunas costeras arenosas. La precipitación (250-380 mm) ocurre durante la estación invernal, en que también son frecuentes las nieblas.

El matorral costero forma un estrato de menor altura $(<1.5 \mathrm{~m})$ y más abierto que el chaparral y frecuentemente contiene un estrato herbáceo. Las especies dominantes son arbustos aromáticos, deciduos facultativos durante el periodo de sequía, de raíces someras y bien adaptados para sobrevivir en una zona de escasa precipitación pero con abundantes nieblas. El follaje grisáceo, flexible y pubescente de muchas de las especies dominantes contrasta marcadamente con los arbustos esclerófilos siempreverdes del chaparral. Plantas significativas de esta región son Artemisia californica, Salvia apiana, Salvia munzii, Eriogonum fasciculatum, Eriophyllum confertiflorum, Rhamnus crocea, Baccharis sarothroides, Hazardia squarrosa var. grindelioides, Opuntia littoralis; y unos pocos arbustos esclerófilos siempreverdes como Rhus integrifolia, Malosma laurina y Simmondsia chinensis.

(iv) Matorral costero rosetófilo.- Esta ecorregión ocupa una franja costera de aproximadamente $175 \mathrm{~km}$ de longitud, desde San Vicente ( $\left.31^{\circ} 20^{\prime} \mathrm{N}\right)$ hasta las proximidades de Punta San Carlos (29 40’ N), formando una larga área transicional entre los ecosistemas mediterráneos y los estrictamente desérticos. Se presenta en las terrazas aluviales y dunas costeras de la vertiente Pacífica de la península. La precipitación (100-250 mm) tiene lugar mayoritariamente durante la estación invernal, aunque pueden caer algunas lluvias estivales. Un significativo aporte de humedad proviene de la niebla costera empujada por los vientos del noroeste del Pacífico.

El matorral costero rosetófilo es más rico en especies que el matorral costero, con las especies suculentas como elemento dominante (especialmente Agavaceae, Cactaceae, Crassulaceae y Euphorbiaceae). Debido a la importancia de la niebla marina como fuente de humedad, los líquenes epífitos son comunes, y plantas con engrosadas hojas suculentas arregladas en rosetas basales son también dominantes. Especies relevantes son Agave shawii, Ambrosia chenopodiifolia, Hazardia rosarica, Hazardia vernicosa, Euphorbia misera, y Dudleya spp. La flora de cactus es muy distintiva: Bergerocactus emoryi, Myrtillocactus cochal, Stenocereus gummosus, Cylindropuntia rosarica y varias especies del 
género Opuntia. Unos pocos árboles deciduos, Aesculus parryi, Fraxinus parryi, y Prunus fremontii, entre otros, pueden aparecer a lo largo de arroyos y cauces.

(v) Islas del Pacífico Norte.- Guadalupe $\left(249 \mathrm{~km}^{2}\right)$ y Cedros $\left(360 \mathrm{~km}^{2}\right)$ son las dos islas de mayor tamaño del conjunto de islas del Pacífico noroeste de Baja California. Su situación geográfica es drásticamente diferente: Guadalupe es una isla oceánica situada a $260 \mathrm{~km}$ de la costa peninsular $\left(29^{\circ} \mathrm{N}\right.$; $\left.118^{\circ} 15^{\prime} \mathrm{W}\right)$, en tanto que Cedros $\left(28^{\circ} 15^{\prime} \mathrm{N} ; 115^{\circ} 15^{\prime} \mathrm{W}\right)$ está tan sólo a $23 \mathrm{~km}$ de la costa de la península del Vizcaíno. Ambas tienen formas ligeramente alargadas, con el eje mayor en disposición aproximada norte-sur, e igualmente presentan una elevada topografía con similares altitudes máximas: 1,295 m para Guadalupe y 1,194 para Cedros.

A pesar de la larga distancia que las separa, tienen un clima oceánico similar. Presentan similares temperaturas anuales promedio $\left(19.9^{\circ} \mathrm{C}\right.$ a nivel del mar en Cedros y $17.7^{\circ} \mathrm{C}$ in Guadalupe) y ambas reciben escasa lluvia, predominantemente en invierno-primavera, y están ampliamente influenciadas por las nieblas costeras.

La precipitación anual promedio medida en la parte baja es de $130 \mathrm{~mm}$ para Guadalupe y $85 \mathrm{~mm}$ para Cedros. El ascenso de la niebla por las pronunciadas pendientes noroccidentales de ambas islas probablemente aumenta la precipitación en las cotas altas. Sin embargo, las frecuentes nieblas son el factor climático dominante en estas islas, complementando la escasa precipitación y permitiendo la presencia de plantas de carácter mediterráneo.

Ambas islas son disyunciones geográficas de la Región Mediterránea, íntimamente relacionadas con la vegetación de las Channel Islands del sur de California y constituyen la expresión insular de la Región Mediterránea. En Guadalupe, el aislamiento ha producido un elevado número de especies de plantas endémicas. La presencia de cabras por más de un siglo ha devastado gran parte de la vegetación original, pero una reciente erradicación está permitiendo cierta recuperación ecológica. En la parte más alta de la isla existe un pequeño bosque del endémico ciprés de Guadalupe (Callitropsis guadalupensis), en tanto que en la punta norte de la isla aproximadamente 130 individuos del pino de Monterrey (Pinus radiata) y unos pocos individuos de encino (Quercus tomentella) sobreviven entre altitudes de 800-1500 m. A elevaciones menores, entre 300-800 m, una comunidad de la endémica palma de Guadalupe (Brahea edulis) persiste en la ladera noroccidental. Una decena de individuos de juníperos (Juniperus californica) sobreviven dispersos en el centro de la isla.

La menos perturbada vegetación del sur de la isla y los pequeños islotes circundantes está caracterizada por especies suculentas, herbáceas perennes como Cistanthe guadalupensis, Stephanomeria guadalupensis, Baeriopsis guadalupensis (un género endémico monotípico), Coreopsis gigantea, y Dudleya guadalupensis. Otras especies en esta comunidad incluyen Hemizonia greeneana, H. palmeri y Perityle incana.
En isla de Cedros la vegetación de la parte baja, que incluiría a los islotes próximos de Islas San Benito, es dominada por matorral desértico con Pachycormus discolor como especie principal, asociada con Agave sebastiana, $\mathrm{Fe}$ rocactus chrysacanthus, Ambrosia chenopodifolia, A. camphorata y Rhus lentii. En los picos más elevados de la isla se observan densos manchones de pino de Monterrey (Pinus radiata), a menor altitud se presentan manchones dispersos de chaparral en las elevadas pendientes del norte de la isla, dominados por el chamizo (Adenostoma fasciculatum), Xylococcus bicolor y Quercus cedrosensis. El junípero (Juniperus californica) se presenta por debajo del cinturón de chaparral, frecuentemente formando un mosaico con matorral costero compuesto de Artemisia californica, Eriophyllum confertiflorum y Eriogonum fasciculatum.

Región desértica (Desierto Sonorense).- Los desiertos de Baja California se extienden a lo largo de la península formando una extensa transición entre los ecosistemas templados de tipo mediterráneo en el noroeste y el extremo sur de la península de carácter tropical. Esta región tiene precipitación escasa e irregular, con variables e impredecibles proporciones de lluvia de invierno y de verano. De manera semejante al Desierto Sonorense continental, y a diferencia de otros desiertos del mundo, la vegetación es caracterizada por una elevada cobertura arbórea. Las ecorregiones son definidas por su situación respecto al eje latitudinal norte-sur, que determina el grado de afectación de las lluvias frontales de invierno desde el norte y los ciclones tropicales en el sur, y por otro lado su respectiva proximidad al Golfo de California o al océano Pacífico, a lo largo de un gradiente esteoeste que determina la temperatura del aire, la influencia de las nieblas costeras y el grado de continentalidad.

La descripción de está región está basada en Shreve (1951), Wiggins (1980), Brown (1994), Peinado et al. (1994a), Delgadillo y Macías-Rodríguez (2002), Peinado et al. (2005; 2006), León de la Luz et al. (2006, 2008).

(i) Desierto de San Felipe o del Bajo Colorado.- El Desierto del Bajo Colorado que se extiende por el noroeste de Sonora, suroeste de Arizona y California se presenta en Baja California como una delgada franja hacia el sur siguiendo la costa norte del Golfo de California. Su límite sur es Bahía de los Ángeles $\left(29^{\circ} \mathrm{N}\right)$, bordeado al oeste por los pronunciados escarpes de las Sierras de Juárez y San Pedro Mártir. Es un área poco montañosa, y está dominada por extensas bajadas aluviales y llanuras de grava y arena. Ubicado en la sombra de lluvia de las elevadas sierras del norte de Baja California, es uno de los desiertos más calientes y secos de Norte América. La temperatura de verano excede los $50{ }^{\circ} \mathrm{C}$ y en las partes más secas la precipitación anual promedia los $50 \mathrm{~mm}$, frecuentemente con varios años consecutivos sin lluvia.

La vegetación es dominada por dos arbustos de hoja reducida y resistentes a la sequía: la gobernadora (Larrea 
tridentata) y la hierba del burro (Ambrosia dumosa), acompañadas por ocotillo (Fouquieria splendens), y agave desértico (Agave deserti). Aunque estas son algunas de las plantas más resistentes a la sequía de Norte América, en las partes más secas de esta ecorregión su presencia está restringida a los cauces de drenaje. A lo largo de arroyos y áreas de piedemonte la vegetación se vuelve más diversa, y algunas leguminosas leñosas como Psorothamnus spinosus, palofierro (Olneya tesota), paloverdes (Parkinsonia florida y P. microphylla) y mesquites (Prosopis pubescens y P. glandulosa var. torreyana) son capaces de establecerse.

(ii) Desierto Central.- Localizado en el parte central de la península, a lo largo de una zona donde las montañas son de moderada elevación y los vientos del Pacífico pueden penetrar tierra adentro, esta ecorregión se extiende desde el Pacífico (entre las latitudes $28^{\circ}$ y $30^{\circ} \mathrm{N}$ ) hasta la prolongada franja costera del Desierto del Bajo Colorado al oriente. En el interior se extiende hacia el norte hasta el piedemonte de las Sierra de San Pedro Mártir y hacia el sur hasta las pendientes de la Sierra de San Francisco.

La precipitación es bi-estacional, pudiendo recibir lluvias frontales en el invierno y precitación ciclónica al final del verano. En las proximidades de la costa Pacífica tiene un clima oceánico moderado con frecuentes nieblas y una precipitación de invierno relativamente regular, en tanto que con la cercanía al Golfo aumenta la temperatura y la proporción de lluvia de verano.

Esta ecorregión, junto con la Costa Central del Golfo, es la que ha proporcionado a Baja California su fama por las bizarras formas arbóreas con grandes y gruesos troncos (plantas sarcocaulescentes), ocasionado por la ocurrencia conjunta del cirio (Fouquieria columnaris), copalquín ( $\mathrm{Pa}$ chycormus discolor), torote (Bursera microphylla), cardón (Pachycereus pringlei), agave costero (Agave shawii), y datilillo (Yucca valida).

Significativamente, las fronteras del Desierto Central pueden delinearse siguiendo la distribución del cirio. Esta es un área única donde elementos de la parte más árida del sur peninsular encuentran su límite norte (ej., Stenocereus gummosus, Myrtillocactus cochal, Pachycereus pringlei, Pachycormus discolor y Yucca valida) y especies del norte alcanzan sus límites meridionales (ej., Fouquieria splendens).

En las proximidades del Pacífico, muchos de los árboles de troncos engrosados de la costa del Golfo pueden verse creciendo en asociación con especies de troncos reducidos y hojas suculentas arregladas en rosetas, capaces de colectar y almacenar agua de las nieblas costeras. Entre ellas destacan algunas especies de Agave, Dudleya y Yucca (Y. valida y Y. whipplei). La influencia de la niebla costera se constata en la abundante presencia de la epífita Tillandsia recurvata y abundantes líquenes.

(iii) Costa Central del Golfo.- Esta ecorregión es una estrecha banda de desierto que se extiende por $800 \mathrm{~km}$ a lo largo de la costa del Golfo, desde Bahía de los Ángeles a lo largo del piedemonte oriental de la Sierra de San Borja hasta la Bahía de La Paz. Las islas de mayor tamaño del Golfo de California, Ángel de la Guarda y Tiburón, así como numerosas islas de menor extensión, junto con $400 \mathrm{~km}$ de banda costera en Sonora pertenecen a estas ecorregión. Su paisaje se caracteriza por colinas desnudas y arroyos bordeados por cantos rodados y depósitos de arena. Su elevación máxima está entre 200-300 m. Es una zona de elevada temperatura y aridez, con precipitación procedente mayoritariamente de tormentas y huracanes del sur al final del verano. La precipitación de invierno es virtualmente nula.

La vegetación es dominada por plantas con troncos gigantes y carnosos, incluyendo al copalquín (Pachycormus discolor) con su corteza anaranjada, torote (Bursera microphylla), copal (B. hindsiana), lomboy (Jatropha cinerea), matacora (J. cuneata), palo blanco (Lysiloma candidum), cardón (Pachycereus pringlei), palo Adán (Fouquieria diguetii), junto con numerosas especies de chollas (Opuntia bigelovii, $O$. cholla, $O$. ramosissima y $O$. tesajo). En las lagunas y humedales costeros se encuentran los manglares más septentrionales con mangle rojo (Rhizophora mangle), mangle negro (Avicennia germinans) y mangle blanco ( $\mathrm{La}$ guncularia racemosa).

(iv) La Sierra de la Giganta.- Esta ecorregión se extiende desde el piedemonte sur del Cerro del Mechudo ( $24^{\circ} 47^{\prime} \mathrm{N}$ ) hasta el Volcán de las Tres Vírgenes $\left(27^{\circ} 30^{\prime} \mathrm{N}\right)$, e incluye todas las áreas montañosas de la Giganta y Guadalupe por encima de $200 \mathrm{~m}$ aproximadamente. Estas sierras forman la columna geológica de la mitad sur de Baja California. La línea de cumbres, con un máximo de altitud de 2,088 m y picos frecuentemente por encima de $800 \mathrm{~m}$, y próxima al Golfo de California, desciende hacia éste de manera escarpada y abrupta. Las pendientes de la vertiente occidental son más suaves y finalmente drenan en las planicies costeras del Pacífico. Torrentes ocasionales pueden arramblar por los cañones de las montañas tras el paso de los huracanes y pueden cambiar dramáticamente el paisaje en cañones y arroyos. En el lado oeste, la topografía más suave mantiene múltiples manantiales y aguajes que alimentan oasis espectaculares. Las temperaturas mensual promedio varía entre 19 y $22{ }^{\circ} \mathrm{C}$. La precipitación tiene lugar principalmente a finales del verano.

La vegetación de esta ecorregión es dominada por gran variedad de leguminosas leñosas como palo hierro (Prosopis palmeri), mesquite dulce ( $P$. glandulosa), palo blanco (Lysiloma candidum), mauto (L. divaricatum), palo hierro (Ebenopsis confinis), vinorama (Acacia brandegeeana), palo chino (A. peninsularis), ojasén (Senna polyantha), y paloverde (Parkinsonia microphylla). Los cactus columnares están pobremente representados, pero la pitahaya dulce (Stenocereus thurberi), Mammillaria spp. y Opuntia spp. son especies comunes. Oasis lineares son característicos de algunos cañones y arroyos, con presencia de palma de abanico (Washingtonia robusta) y palma de taco (Brahea brandegeei). 
ECorRegiones de la PENínsula de Baja CALIFornia: Una síntesis

(v) Desierto de Vizcaíno.- El Desierto de Vizcaíno está formado por una serie de extensas llanuras áridas, por debajo de $100 \mathrm{~m}$ de altitud, que se extienden a lo largo de la vertiente del Pacífico entre $26^{\circ}$ y $29^{\circ} \mathrm{N}$. Contiene extensas llanuras desérticas, dunas interiores y suelos salinos. Las únicas elevaciones ocurren en el margen occidental de la península de Vizcaíno, donde las pequeñas montañas de la Sierra del Placer y los Picachos de Santa Clara (700 m) capturan la niebla y humedad procedente del océano. La influencia marina procedente del Pacífico produce un clima fresco, nuboso y ventoso la mayor parte del año, con frecuentes mañanas de niebla. Recibe alrededor de $100 \mathrm{~mm}$ de lluvia, concentrada principalmente en invierno, pero tan baja precipitación es compensada parcialmente por las frecuentes nieblas y los periodos de nubosidad que reducen la evaporación. La aridez y la brisa salina mantiene una vegetación de reducida riqueza caracterizada por unos pocos individuos miniaturizados, frecuentemente postrados, de arbustos perennes como yuca (Yucca valida), palo Adán (Fouquieria diguetti) y lomboy (Jatropha cinerea) en las partes menos áridas, pero las extensas llanuras alcalinas están dominadas por Atriplex julacea y Frankenia palmeri, acompañadas por otros arbustos tolerantes a la salinidad como Atriplex polycarpa, A. canescens ssp. linearis, Encelia farinosa, Viguiera deltoidea, V. microphylla y Lycium californicum.

(vi) Llanos de Magdalena- Esta región ocupa las tierras bajas de suaves pendientes y el drenaje Pacífico del Corredor de La Giganta, extendiéndose desde la Bahía de San Juanico $\left(26^{\circ} 15^{\prime} \mathrm{N}\right)$ en el norte hasta la ciudad de Todos Santos en el sur. Topográficamente, contiene dos secciones bien diferenciadas: una porción oriental compuesta por las colinas y mesas volcánicas a lo largo de los piedemonte de las Sierras de Guadalupe y La Giganta, y una occidental de extensas llanuras arenosas de escasa elevación que bordean el océano Pacífico. La precipitación principalmente proviene de los huracanes de final de verano, pero ocasionalmente llegan lluvias de invierno. Al igual que en el Desierto de Vizcaíno, el clima está fuertemente influido por la corriente de California y las surgencias frías asociadas. La niebla matutina es frecuente durante gran parte del año, promoviendo abundante crecimiento de bromelias epífitas como los gallitos (Tillandsia recurvata y $T$. ferrisiana) y líquenes como la orchilla (Ramalina menziesii) que cubren las plantas en las cercanías de la costa.

La proximidad a los matorrales secos tropicales que cubren la punta sur de la península es evidente en esta región. La densidad de suculentas en roseta es menor, y los árboles del desierto coexisten con cactus columnares gigantes. Los torotes (Bursera filicifolia, B. hindsiana, y B. microphylla), mesquite (Prosopis glandulosa), palo Adán (Fouquieria diguetii), paloverde (Parkinsonia florida), ciruelo (Cyrtocarpa edulis), y palo blanco (Lysiloma candidum) forman densos matorrales en algunos de los arroyos. Los cactus gigantes como el cardón (Pachycereus pringlei), pitahaya agria (Ste- nocereus gummosus), senita (Lophocereus schottii), y chollas (Cylindropuntia spp.) son comunes en las extensas llanuras. Una de las plantas más características de esta región es la chirinola (Stenocereus gummosus), un cactus columnar endémico de las planicies costeras de los Llanos de Yrais, que sorprendentemente crece de manera rastrera.

Región tropical (Región del Cabo).- La región tropical seca ocupa el extremo sur de la península de Baja California. Es cruzada por el Trópico de Cáncer $\left(23^{\circ} 27^{\prime} \mathrm{N}\right)$, y al igual que los ecosistemas mediterráneos en el norte, recibe mayor precipitación que los desiertos peninsulares. Sin embargo, la precipitación en la esta región es mayoritariamente derivada de tormentas ciclónicas tropicales que alcanzan la región a finales de verano y otoño. La Sierra de la Laguna, una espectacular cadena montañosa granítica que alcanza los 2,090 m de altitud, atraviesa la Región del Cabo de norte a sur y ha dado origen a la mayoría de los suelos de esta región.

La descripción de está región está basada en Shreve (1937), Wiggins (1980), Arriaga y Ortega (1988), Arriaga y León de la Luz (1989), León de la Luz y Domínguez-Cadena (1989) León de la Luz et al. (2000).

(i) Matorral tropical del Cabo.- Esta ecorregión comprende las tierras bajas situadas al este y sur de la falla de La Paz, una línea suavemente arqueada que discurre desde la Ensenada del Coyote en el Golfo de California hasta Todos Santos en la costa Pacífica. Esta línea geológica divide las formaciones graníticas de la Región del Cabo de los basaltos volcánicos de la Giganta, y marca la frontera entre los ecosistemas tropicales secos y los estrictamente desérticos en la península.

Ocupa un paisaje característico como las llanuras aluviales costeras, desde el nivel del mar hasta aproximadamente $500 \mathrm{~m}$ de altitud. Con una precipitación anual menor a 200 $\mathrm{mm}$, de origen monzónico o de esporádicas tormentas tropicales, están cubiertas por un matorral bajo de troncos carnosos (sarcocaulescente), con mayor riqueza de especies y endemismo que los matorrales desérticos. Plantas semi-suculentas como el torote (Bursera microphylla), lomboy ( $\mathrm{Ja}$ tropha cinerea), matacora (J. cuneata), ciruelo (Cyrtocarpa edulis); especies arborescentes como paloverde (Parkinsonia florida subsp. peninsulare), cacachila (Karwinskia humboldtiana), Colubrina triflora, higuera (Ficus brandegeei), palo chino (Havardia mexicana), palo Adán (Fouquieria diguetii), palo amarillo (Esenbeckia flava), mesquite (Prosopis articulata); y elementos suculentos como el cardón barbón (Pachycereus pecten-aboriginum), cardón (P. pringlei) y cholla pelona (Opuntia cholla) son algunas de las plantas más comunes en este paisaje.

(ii) Selva baja del Cabo.- El piedemonte de las montañas de la Región del Cabo entre 500 y 1,000 de altitud contiene los únicos bosques tropicales secos de Baja California, que permanecen sin hojas nueves meses al año y rebrotan durante la temporada de lluvias. Aunque las lluvias monzónicas de ve- 
rano pueden alcanzar esta región, la mayor parte de la precipitación proviene de los ciclones y tormentas tropicales que se forman en el Pacífico tropical y proveen un promedio de 200-400 mm de precipitación anual. La temperatura promedio anual varía entre 22 y $24^{\circ} \mathrm{C}$ y las heladas están ausentes.

La flora de esta comunidad es muy rica en especies, algunas especies típicamente perennes como palo blanco (Lysiloma divaricatum), jacolosúchil (Plumeria acutifolia), cardón barbón (Pachycereus pecten-aboriginum), flor de San José (Senna atomaria), colorín (Erythrina flabelliformis), palo ébano (Chloroleucon mangense), ocote (Gochnatia arborescens), torote (Bursera cerasifolia), guayparín (Diospyros californica), Sebastiania pavoniana, encino negro (Quercus brandegeei), palo escopeta (Albizia occidentalis), lomboy colorado (Jatropha vernicosa), y naranjillo (Zanthoxylun arborescens).

(iii) Bosque de la Sierra de La Laguna.- En las altas elevaciones de la Sierra de La Laguna, a altitudes superiores a $1,000 \mathrm{~m}$, se encuentra una densa masa forestal, ocupando una relativamente pequeña área de unos $500 \mathrm{~km}^{2}$. Las montañas, formadas mayoritariamente de granito y otras rocas intrusivas, se elevan en pronunciadas y escarpadas pendientes hasta los 2,090 m. La precipitación ocurre principalmente de Agosto a Octubre y originada por los ciclones tropicales del Pacífico, y alcanza un promedio anual de $700 \mathrm{~mm}$.

Debido a su larga historia de aislamiento evolutivo, esta ecorregión muestra altos niveles de endemismo, con aproximadamente el $15 \%$ de las plantas exclusivas de esta área. Las escarpadas pendientes de las altitudes medias $(1,000$ $1,500 \mathrm{~m}$ ) están cubiertos de bosques abiertos de encino, donde predominan individuos dispersos de encino roble (Quercus tuberculata), creciendo en medio de arbustos de baja estatura como la celosa (Mimosa xanti), vinorama (Acacia brandegeeana), granadina (Dodonaea viscosa), Tephrosia cana, y bernardia (Bernardia lagunensis). La parte elevada (1,500-2,000 m) está ocupada por bosque de pino-encino, donde las especies dominantes son el pino piñonero (Pinus lagunae), encino negro (Quercus devia), madroño (Arbutus peninsularis) y sotol (Nolina beldingi).

\section{Agradecimientos}

El Grupo de Trabajo de Ecorregiones está integrado por: Mike Allen y Cameron Barrows de la University of California-Riverside, José María Beltrán de Pronatura-Noroeste, José Delgadillo de la Facultad de Ciencias, Universidad Autónoma de Baja California, Richard S. Felger de The University of Arizona Herbarium, Ernesto Franco-Vizcaíno del CICESE, Anne Gondor de The Nature Conservancy, Alejandro Hinojosa del CICESE, José Luis León de la Luz del Centro de Investigaciones Biológicas del Noroeste, Erik Mellink del CICESE, Scott Morrison, Thomas A. Oberbauer del Department of Planning and Land Use, Kristine Preston de la University of California-Riverside, John Rotenberry de la University of California-Riverside, Mario Salazar del CICESE, Sula Vanderplank del Rancho Santa Ana Botanic Garden. Estamos en deuda con la Universidad Autónoma de Baja California y a José Delgadillo por el espacio y facilidades brindadas durante el taller, así como a Sarahuén Ávila y Francisco Casillas por la ayuda durante el desarrollo del mismo. Los comentarios y sugerencias realizados por la Dra. Victoria Sosa, Hugo Tovar y por dos revisores anónimos mejoraron sustancialmente el presente trabajo. Este trabajo fue financiado por Alcoa Foundation y el Museo de Historia Natural de San Diego.

\section{Literatura citada}

Aguirre-L. G., Morafka D.J., y Murphy R.W. 1999. The peninsular archipelago of Baja California: a thousand kilometers of tree lizard genetics. Herpetologica 55:369-381

Álvarez-Castañeda S.T. y Patton J.L. Eds. 1999. Mamíferos del noroeste de México. Centro de Investigaciones Biológicas del Noroeste, La Paz, B.C.S.

Álvarez-Castañeda S.T., Salinas-Zavala C.A. y de Lachica, F. 1995. Análisis biogeográfico del noroeste de México con énfasis en la variación climática y mastozoológica. Acta Zoologica Mexicana 66:59-86.

Arriaga L. y Ortega A. Eds. 1988. La Sierra de La Laguna de Baja California Sur. Publ. No. 1, CIBNOR, La Paz.

Arriaga L. y León de la Luz J.L. 1989. The Mexican tropical deciduous forest of Baja California Sur: a floristic and structural approach. Plant Ecology 84:45-52.

Arriaga L., Aguilar C., Espinosa D. y Jiménez R. Eds. 1997. Regionalización ecológica y biogeográfica de México. Comisión nacional para el conocimiento y uso de la biodiversidad, México D.F.

Aschmann H. 1959. The Central Desert of Baja California: Demography and ecology. University of California Press., Berkeley y Los Angeles, CA.

Bailey R.G. 1983. Delineation of ecosystem regions. Environmental Management 7:365-373.

Bailey R.G. 2004. Identifying ecoregion boundaries. Environmental Management 34:S14-S26.

Bancroft G. 1926. The faunal areas of Baja California Norte. The Condor 28:209-215.

Brandegee T.S. 1891. Flora of the Cape Region of Baja California. Proceedings of the California Academy of Sciences Serie 2: $108-182$

Brandegee T.S. 1892. The distribution of the Flora of the Cape Region of Baja California. Zoe 3: 223-231.

Breceda A., León de la Luz J.L. y Arriaga L. 1994. Phytogeographic relationships of the Tropical Dry Forest of Baja California Sur with the Sonoran Desert and the nearest tropical vegetation of mainland Mexico. Memoires de la Societe de Biogegographie, Sér. 3, 4:45-52.

Brown J.W., Real H.G. y Faulkner, D.K. 1992. Butterflies of Baja California. The Lepidoptera Research Foundation, Inc. Beverly Hills, CA.

Brown D.E. Ed. 1994. Biotic Communities: Southwestern United States and Northwestern Mexico. University of Utah Press, Salt Lake City, Utah.

Crum H. y Miller H.A. 1956. Bryophytes from Guadalupe Island, 
Baja California. The Southwestern Naturalist 1:116-120.

Cummins K.W. 1974. Structure and function of stream ecosystems. Bioscience 24:631-641.

Daniel T. 1997. The Acanthaceae of California and the peninsula of Baja California. Proceedings of the California Academy of Sciences 49:309-403.

del Barco, Miguel. 1780 ca. Correcciones y adiciones a la historia o noticia de la California en su primera edición de Madrid, año de 1757. (editado por M. León-Portilla como Historia Natural y Crónica de la antigua California; Universidad Nacional Autónoma de México, Instituto de Investigaciones Históricas, 1988; México, D.F.).

Delgadillo J. 1998. Florística y ecología del norte de Baja California. Universidad Autónoma de Baja California, Ensenada.

Delgadillo J. 2004. El bosque de coníferas de la Sierra de San Pedro Mártir, Baja California. SEMARNAT - Instituto Nacional de Ecología, México DF.

Delgadillo J. y Macías-Rodríguez M.A. 2002. Componente florístico del desierto de San Felipe, Baja California, México. Boletín de la Sociedad Botánica de México 70:45-65.

Eisen G. 1897. Explorations in the Cape Region of Baja California. Journal of American Geographical Society of New York 29:271-280.

Eisen G. 1900. Explorations in Central Baja California. Journal of the American Geographical Society of New York 32:397-429.

Epling C. y Lewis H. 1942. The centers of distribution of the chaparral and coastal sage associations. American Midland Naturalist 27:445-462.

Faulkner D. 1982. The endemic butterflies of Baja California. Environment Southwest 497:9-13.

Garcillán P.P. y Ezcurra E. 2003. Biogeographic regions and $\beta$-diversity of woody dryland legumes in the Baja California peninsula. Journal of Vegetation Science 14:859-868.

Garcillán P.P., Ezcurra E. y Riemann H. 2003. Distribution and species richness of woody dryland legumes in Baja California, México. Journal of Vegetation Science 14:475-486.

Garcillán P.P., González-Abraham C.E. y Ezcurra E. 2010. The cartographers of life: Two centuries of mapping the natural history of Baja California. Journal of the Southwest 52:1-40

Gentry H.S. 1978. The agaves of Baja California. California Academy of Sciences, San Francisco, CA.

Grime J.P., Hodgson J.G. y Hunt R. 1988. Comparative plant ecology: A functional approach to common British species. Unwin Hyman, London.

Grinnell J. 1928. A distributional summation of the ornithology of Lower California. University of California Publications in Zoology 32:1-300.

Grismer L.L. 1994. Ecogeography of the peninsular herpetofauna of Baja California, Mexico, and its utility in historical biogeography. En: Wright J.W. y Brown P. Eds. Herpetology of the North American deserts, pp.89-125. Southwestern Herpetologists Society, Van Nuys.

Grismer L.L. 2002. Amphibians and reptiles of Baja California, including its Pacific islands and the islands in the Sea of Cortés. The University of California Press, Berkeley, CA.

Hastings J.R. Tyurner R.M. 1965. Seasonal precipitation regimes in Baja California, Mexico. Geografiska Annaler. Series A, Physical Geography 47:204-223.

Herrera C.M. 1984. Tipos morfológicos y funcionales en plantas del matorral mediterráneo del sur de España. Stvdia Oecologica

\section{5:7-34.}

Hijmans R.J., Cameron S.E., Parra J.L., Jones P.G. y Jarvis A. 2005. Very high resolution interpolated climate surfaces for global land areas. International Journal of Climatology 25:19651978.

INEGI [Instituto Nacional de Geografía y Estadística]. 1990. Provincias fisiográficas de México, escala 1:4,000,000.

INEGI. 1995. Mapa digital de edafología de Baja California y Baja California Sur, escala 1:250,000.

INEGI. 1997. Mapa digital de uso de suelo y vegetación de Baja California y Baja California Sur (Serie 2), escala 1:250,000.

INEGI. 1998. Curvas de nivel de Baja California y Baja California Sur, escala 1: 250,000.

INEGI. 2004. Mapa digital de geología de Baja California y Baja California Sur, escala 1:250,000.

Koch L.F. y Crum H.A. 1950. Mosses of Baja California. Rev. Bryol. et Lichenol. 19:188-192.

León de la Luz J.L. y Domínguez Cadena R. 1989. Flora of the Sierra de la Laguna, Baja California Sur, Mexico. Madroño 36:61-83.

León de la Luz J.L., Pérez-Navarro J.J. y Breceda A. 2000. A transitional xerophytic tropical plant community of the Cape Region, Baja California. Journal of Vegetation Science 11:555564.

León de la Luz J.L. y Domínguez-Cadena R. 2006. Hydrophytes of the oases in the Sierra de la Giganta of Central Baja California Sur, Mexico: Floristic composition and conservation status. Journal of Arid Environments 67:553-565.

León de la Luz J.L., Rebman J., Domínguez-León M. y Domínguez-Cadena R. 2008. The vascular flora and floristic relationships of the Sierra de La Giganta in Baja California Sur, Mexico. Revista Mexicana de Biodiversidad 79:29-65.

Lomolino M.V., Riddle B.R. y Brown J.H. 2006. Biogeography. Sinauer Associates, Inc., Sunderland, Massachusetts.

McGinnies W.G. 1981. Discovering the desert: legacy of the Carnegie Desert Botanical Laboratory. University of Arizona Press, Tucson.

Mellink E., 2002. El límite sur de la región mediterránea de Baja California, con base en sus tetrápodos endémicos. Acta Zoologica Mexicana 85:11-23.

Minnich R.A. y Franco-Vizcaíno E. 1998. Land of Chamise and Pines: historical accounts and current status of northern Baja California's vegetation. University of California Press, Berkely y Los Ángeles, CA.

Minnich R.A. y Franco-Vizcaíno E. 1999. La vegetación mediterránea de Baja California. Fremontia Edición especial 4-15.

Miranda F. y Hernández-X. E.1963. Los tipos de vegetación de México y su clasificación. Boletín de la Sociedad Botánica de México 28:29-179.

Mooney H.A. y Harrison A.T. 1972. The vegetation gradient on the lower slopes of the Sierra San Pedro Mártir in northwest Baja California. Madroño 21:439-445.

Moran R. 1996. The flora of Guadalupe Island, Mexico. Memoirs of the California Academy of Sciences, No. 19. California Academy of Sciences, San Francisco, CA.

Murphy R.W. 1983. Paleobiogeography and genetic differentiation of the Baja California herpetofauna. Occasional papers of the California Academy of Sciences 137:1-48.

Nelson E.W. 1921. Lower California and its natural resources. National Academy of Sciences, Riverside, CA. 
Oberbauer T. 1993. Floristic analysis of vegetation communities on Isla de Cedros, Baja California, Mexico. En: Hochberg F.G. Ed. Third California Island Symposium: recent advancees in research on the California Islands, pp.115-131, Santa Barbara Museum of Natural History, Santa Barbara, CA.

Omernik J.M. 1987. Map supplement: Ecoregions of the conterminous United States. Annals of the Association of American Geographers 77:118-125.

Peinado M., Alcaraz F., Delgadillo J. y Aguado I. 1994a. Fitogeografía de la península de Baja California, México. Anales Jardín Botánico de Madrid 51:255-277.

Peinado M., Alcaraz F., Aguirre J.L. y Álvarez, J. 1994b. Vegetation formations and associations of the zonobiomes along the North American Pacific coast. Vegetatio 114:123-135.

Peinado M., Alcaraz F., Aguirre J.L., Delgadillo J. y Aguado I. 1995. Shrubland formations and associations in mediterraneandesert transitional zones of northwestern Baja California. Vegetatio 117:165-179.

Peinado M., Delgadillo J. y Aguirre, J.L. 2005. Plant associations of El Vizcaíno biosphere reserve, Baja California Sur, Mexico. The Southwestern Naturalist 50:129-149.

Peinado M., Macías M., Delgadillo J. y Aguirre J.L. 2006. Major plant communities of North America's most arid region: The San Felipe Desert, Baja California, México. Plant Biosystems 140:280-296.

Peinado M., Aguirre J., Delgadillo J. y Macías M.A. 2008. A phytosociological and phytogeographical survey of the coastal vegetation of western North America. Part I: plant communities of Baja California, Mexico. Plant Ecology 196:27-60.

Raunkiaer C. 1934. The life forms of plants and statistical plant geography. Oxford University Press, Oxford.

Raven P.H. y Axelrod D.I. 1978. Origin and relationships of the California flora. University of California Publications in Botany 72, University of California Press, Berkely y Los Angeles, CA.

Riddle B.R., Hafner D.J., Alexander L.F. y Jaeger J.R. 2000. Cryptic vicariance in the historical assembly of a Baja California Peninsular Desert biota. Proceedings of the National Academy of Sciences 97:14438-14443.

Rojas-Soto O.R., Alcántara-Ayala O. y Navarro A.G. 2003. Regionalization of the avifauna of the Baja California peninsula, Mexico: a parsimony analysis of endemicity and distributional modelling approach. Journal of Biogeography 30:449-461.

Root R.B. 1967. The Niche exploration pattern of a blue grey gna- tcatcher. Ecological Monographs 37:317-350.

Rowe J.S. y Sheard J.W. 1981. Ecological land classification: a survey approach. Environmental Management 5:451-464.

Rzedowski J. 1978. Vegetación de México. Limusa. México D.F.

San Diego Natural History Museum. The Flora of Baja California

<http://www.bajaflora.org> (Revisado en Julio-Dic 2006, y durante 2007 a 2010).

Shreve F. 1936. The transition from desert to chaparral in Baja California. Madroño 3:257-264.

Shreve F. 1937. The vegetation of the Cape Region of Baja California. Madroño 4:105-136.

Shreve F. 1951. Vegetation of the Sonoran Desert. Carnegie Institution of Washington, Washington, D.C.

Smith A.G., Miller W.B., Christensen C.C. y Roth B. 1990. Land mollusca of Baja California, Mexico. Proceedings of the California Academy of Sciences 47:95-158.

Stebbins G.L. 1974. Flowering plants: Evolution above the species level. The Belknap Press of Harvard University Press, Massachusetts.

Thorne R.F. 1969. The California Islands. Annals of the Missouri Botanical Garden 56:391-408.

Turner R.M., Bowers J.E. y Burgess T.L. 1995. Sonoran Desert Plants. An Ecological Atlas. The University of Arizona Press, Tucson, Arizona.

Upton D.E. y Murphy R.W. 1997. Phylogeny of the side-blotched lizards (Phrynosomatidae: Uta) based on mtDNA sequences: support for a midpeninsular seaway in Baja California. Molecular Phylogenetics and Evolution 8:104-113

Wallace G.D. 1985. Vascular plants of the Channel Islands of southern California and Guadalupe Island, Baja California, Mexico. Contributions in Science 365. Natural History Museum Los Angeles Co., Los Angeles, CA.

Westman W.E. 1983. Xeric Mediterranean-type shrubland associations of Alta and Baja California and the community/continuum debate. Vegetatio 52:3-19.

Wiggins I. 1980. Flora of Baja California. Stanford University Press, Stanford, CA.

Zink R.M. 2002. Methods in comparative phylogeography, and their application to studying evolution in the North American aridlands. Integrative and Comparative Biology 42:953-959

Zippin D.B. y Vanderwier J.M. 1994. Scrub community descriptions of the Baja California penisnula, Mexico. Madroño 41:85-119.

Recibido: 16 de julio de 2010

Aceptado: 12 de noviembre de 2010 